\title{
Avobenzone, Guaiazulene and Tioxolone identified as potent autophagy inducers in a high-throughput image based screen for autophagy flux
}

Surendra Kumar Prajapat, Chandru Subramani ${ }^{1}$, Sudhanshu Vrati \& Manjula Kalia*

Regional Centre for Biotechnology, NCR Biotech Science Cluster, Faridabad, 121001,

Haryana, India

*Correspondence: Manjula Kalia, manjula@rcb.res.in

${ }^{1}$ Present address: Department of Pathology, University of Texas medical branch, Galveston,

Texas 77550 


\begin{abstract}
Autophagy is a conserved intracellular degradation pathway that is essential for maintaining cellular homeostasis. Given its critical role in several disease conditions, recent studies are focussed on identifying drugs/small molecules with autophagy modulating capacity for potential clinical applications. Here, we describe the development and characterisation of a quantitative image-based high content screening platform for autophagy flux measurements using the human melanoma A375 cell line that stably expresses the GFP-LC3RFP probe. The GFP-LC3 is incorporated into autophagosomes, while RFP serves an internal control. The GFP/RFP fluorescence intensity ratio gives an accurate indication of autophagy induction (low ratio) vs blockage of autophagy flux (high ratio), and was validated with the autophagy inducer Torin1 and inhibitor Bafilomycin A1. This assay was used to screen the Spectrum collection library comprising of 2560 compounds, to identify autophagy modulators. In addition to known autophagy effectors, several novel autophagy inducers and inhibitors were identified in our study. Further three FDA approved drugs that are widely used in skin-care products: Avobenzone, Guaiazulene and Tioxolone, were validated as potent autophagy inducers that function in an mTOR independent manner.
\end{abstract}

Keywords: Autophagy; Avobenzone; Guaiazulene; Tioxolone; high content screening; Microsource spectrum library

Abbreviations: Baf-A1, Bafilomycin A1; LC3, Microtubule-associated protein light chain 3; mTOR, mechanistic target of rapamycin, 


\section{Introduction}

Macroautophagy (hereafter, autophagy) is an intracellular degradative pathway, conserved from yeast to human. It occurs at a basal level to maintain cellular homeostasis, and is upregulated during starvation and disease conditions [1]. Recent studies have shown that pharmacological modulation of autophagy holds tremendous therapeutic potential for disease conditions such as neurodegeneration and cancer [2], and hence identification of autophagy modulating drugs/compounds is an active area of research [3].

The measurement of autophagy is done by monitoring the lipidated levels of microtubuleassociated protein light chain 3 (LC3) protein that specifically incorporates into autophagosomes. Other techniques involve direct visualization of autophagosomes through fluorescence or electron microscopy. The degradative capacity of the pathway or autophagy flux is analysed by using specific inhibitors of autophagosome-lysosome fusion either through western blotting for LC3-II levels or by using ratiometric fluorescence based assays [4-6]. These have been further developed for high throughput platforms and can enable testing the autophagy capacity of several thousand compounds. A series of image based high throughput screening studies using probes such as mRFP-GFP-LC3, GPF-LC3-RFP-LC3 $\triangle$ G, GFP-LC3-RFP etc. and have identified autophagy inducers with broad translation potential [6-10].

Here we have developed an image-based high content quantitative assay using the human melanoma A375 cell line stably expressing the fluorescent probe GFP-LC3-RFP [6]. In a primary screening of the Microsource spectrum library, several autophagy flux inducers and inhibitors were identified by low and high GFP/RFP signal ratios respectively. Three drugs widely used in skin-care products Avobenzone, Guaiazulene and Tioxolone, were further validated for their autophagy inducing properties. 


\section{Materials and Methods}

\section{Cell lines}

A375 and HEK293T cells were obtained from the Cell Repository at the National Centre for Cell Sciences, Pune, India. Cells were grown in Dulbecco's modified Eagle's medium (DMEM) (AL007A, HiMedia) supplemented with 10\% fetal bovine serum (FBS) (RM10432, HiMedia), $100 \mu \mathrm{g} / \mathrm{ml}$ penicillin-streptomycin, $2 \mathrm{mM}$ L-glutamine and 1x MEM Non-Essential Amino Acids Solution (ACLO06, HiMedia).

\section{Reagents, antibodies and plasmids}

The following reagents were used in the study: Torin1 (4247, Tocris), Bafilomycin (Baf)-A1 (B1793-10UG, Sigma), PMSF (P7626-100G, Sigma), Protease inhibitor cocktail (11697498001, Roche). The Spectrum collection library (SP170615, MicroSource Discovery Systems, Inc.) comprising of 2560 compounds including bioactive molecules, natural products, and FDA-approved compounds was used for image based high content screening. The following antibodies were used in the study: GAPDH (14C10); Phospho-4E-BP (Thr37/46) (2855S); 4E-BP (9644S); Phospho-p70 S6 Kinase (Thr389) (97596S) and p70 S6 Kinase (2708S) from Cell Signalling Technology, LC3 (ab51520); RFP (ab62341) and GFP (ab32146) from Abcam, HRP-conjugated secondary antibodies from Jackson ImmunoResearch Laboratories. The following plasmids were obtained from Addgene: gag/pol (\#14887) [11], pCI-VSVG (\#1733) [12], and pMRX-IP-GFP-LC3-RFP (\#84573) [6].

\section{Stable cell line generation}

HEK293T cells were co-transfected with gag/pol, pCI-VSVG and pMRX-IP-GFP-LC3-RFP plasmids using Lipofectamine 2000 and incubated at $37^{\circ} \mathrm{C}$ for $24 \mathrm{~h}$. The supernatant was harvested for retrovirus, and was used to transduce A375 cells. Puromycin selection (2 $\mu \mathrm{g} / \mathrm{ml}$ ) was given after $48 \mathrm{~h}$. After one week of selection, cells were trypsinized and 10,000 
cells were serially diluted to achieve single cell seeding per well of a 96 well-plate. Isolated colonies were visible by 14 days, and were tested for the expression of GFP, RFP and autophagy flux using Torin1 and Baf-A1 treatments. One clone was chosen for further studies.

\section{Drug treatment \& confocal imaging}

For confocal microscopy GFP-LC3-RFP A375 cells were grown on glass coverslips, and were treated with DMSO (control), Torin1 $(1 \mu \mathrm{M})$ or Baf-A1 (100 nM) for $6 \mathrm{~h}$. Images were acquired on FV3000 confocal microscope using 60X objective. GFP/RFP intensity ratio per cell was calculated and normalized to DMSO control treated cells.

\section{High content imaging and analysis}

For image based high content screening 15,000 cells/well were seeded in Corning 96 well black polystyrene microplates (CLS 3603). Torin1 and Baf-A1 treatment was given for $6 \mathrm{~h}$, while drug treatment was given at $10 \mu \mathrm{M}$ for $12 \mathrm{~h}$. All drugs were tested in biological duplicates or triplicates. Cells were fixed and stained with DAPI. Images were acquired on ImageXpress Micro Confocal High-Content Imaging System (Molecular devices, USA) using FITC, Texas red, and DAPI channels with a $10 \mathrm{X}$ objective lens. A total of 16 fields per well were acquired and this covered the entire well area. Analysis was performed using the multi-wavelength cell scoring module of the MetaXpress Software. To calculate drug cytotoxicity, total nuclei were counted using DAPI in drug and DMSO treated wells. Any drug showing more than $20 \%$ reduction in total nuclei was considered as toxic and excluded from further analysis. Only triple positive cells (DAPI, GFP, RFP) were used for quantitation. The integral intensity of GFP and RFP per well was calculated and used to estimate the GFP/RFP ratio. The values obtained for DMSO treatment were used for normalization. The GFP/RFP ratio in Torin 1 treatment gave a mean value of 0.6 , while BafA1 treatment gave a mean 
value of 1.2. Any drug showing a GFP/RFP ratio $<0.8$ was considered as an autophagy inducer, and GFP/RFP ratio $>1.2$ was marked as autophagy flux inhibitor.

\section{Western blotting}

After drug treatment A375 cells were washed with PBS and lysed using cell lysis buffer [150 $\mathrm{mM} \mathrm{NaCl}, 1 \%$ Triton X-100, $50 \mathrm{mM}$ Tris- $\mathrm{HCl}$ (pH 7.5), $200 \mu \mathrm{M}$ PMSF and protease inhibitor cocktail]. Protein quantification was done using BCA assay (786-570, G-Bioscience) kit. Cell lysates were separated on polyacrylamide gels and transferred to a PVDF membrane for immunoblotting using specific primary and secondary antibodies. Western blot images were processed using Image ( $\mathrm{NIH}$, USA) software for quantitation of band intensities. Data are represented as mean \pm SD from three independent experiments.

\section{Cell viability assay}

A375 cells were seeded at a density of 15000 cells/well in 96-well plates. After $24 \mathrm{~h}$, cells were treated with Avobenzone/ Guaiazulene Tioxolone $(10 \mu \mathrm{M})$ for $12 \mathrm{~h}$. The cytotoxicity assay was performed using CellTiter-Glo kit (G7572, Promega). Percentage of cell viability was measured relative to DMSO treated cells.

\section{Statistical analysis}

Statistical analysis of the data was performed using paired Student $t$-test and differences were considered significant at values of $* P<0.05, * * P<0.01, * * * P<0.001$. 


\section{Results}

\section{Generation and validation of $A 375$ cell line stably expressing autophagy flux probe}

Studies have established that the GFP-LC3-RFP probe is a powerful tool for measurements of autophagy flux $[5,6,13]$. This protein is cleaved by the cellular cysteine protease ATG4, into two equimolar fragments of GFP-LC3 and RFP. The GFP-LC3 is lipidated with phosphatidylethanolamine (PE) at the terminal glycine residue, resulting in its incorporation into autophagosomes. Depending on the status of autophagy in the cell, the levels of GFPLC3 change, while the RFP serves as an internal control (Fig 1A). The ratiometric measurements of GFP/RFP relative to a basal/untreated condition are an accurate reflection of autophagy induction or inhibition in the cell (Fig 1A) $[6,14]$.

We generated a stable human melanoma A375 cell line expressing this autophagy flux probe (Fig 1B). Positive clones were validated, and one clone for chosen for further studies. The GFP-LC3 expression was detected using both GFP and LC3 antibodies (Fig 1B). Roughly equal levels of RFP were also seen (Fig 1B). Fluorescence images showed that the clone expressed GFP-LC3 that showed punctate distribution in the cell corresponding to autophagosomes, while diffuse RFP staining was observed throughout the cytosol (Fig 1C, upper panel). Treatment of these cells with Torin1, a potent autophagy inducer resulted in a clear reduction in GFP-fluorescence in these cells, while treatment with Baf-A1 which inhibits autophagosome-lysosome fusion showed enhanced GFP intensity (Fig 1C). GFP/RFP ratios in the Torin1 and Baf-A1 treated cells were normalized to DMSO treated cells. Torin1 treatment showed a clear reduction of GFP/RFP ratio indicative of high autophagy flux, while Baf-A1 showed an enhanced ratio indicative of low autophagy flux (Fig 1D). These data established the validity of this cell line for autophagy flux measurements.

\section{Establishment of a high content screening platform}


This cell line was next tested in a high throughput 96 well plate platform. Cells were treated with DMSO (control) or Torin1/ Baf-A1, and images were acquired. As seen earlier through confocal microscopy, Torin1 and Baf-A1 treatment resulted in reduced and enhanced GFP/RFP ratios respectively (Fig 2A). Based on values obtained with Torin1 and Baf-A1, we established a cut off limit of $<0.8$ for autophagy inducers and $>1.2$ for autophagy inhibitors (Fig 2B). Using this technique, we screened the Spectrum library of 2560 compounds at 10 $\mu \mathrm{M}$ concentration. The cytotoxicity of the drug treatment was established through DAPI staining compared to untreated controls as described in the methods section (Data Sheet S1). Based on the GFP/RFP ratios, we identified 104 drugs that changed the cellular autophagy levels (Fig 1C, File S1). Of these 53 drugs have been previously established as autophagy inducers in literature $[6,7,9]$. We further identified 31 novel autophagy inducers and 20 novel autophagy inhibitors (File S1).

\section{Avobenzone, Guaiazulene and Tioxolone identified as potent autophagy inducers}

Of the shortlisted drugs, we focused our attention on three FDA-approved drugs that are widely used in skin-care products: Avobenzone, Guaiazulene and Tioxolone. All the drugs were retested for toxicity (Fig 3A), and showed GFP/RFP ratios comparable to Torin1 (Fig 3B, C), identifying them as potent autophagy inducers. We further validated autophagy induction through western blotting for LC3. All the drugs showed enhanced LC3-II levels (Fig 3D). By using Baf-A1 we confirmed that LC3-II accumulation caused by these drugs was due to autophagy induction and not due to blockage of autophagosome-lysosome fusion (Fig 3F). We also tested if these drugs acted in an mTOR dependent manner by checking phosphorylation levels of S6 kinase (S6K) and eukaryotic translation initiation factor 4Ebinding protein 1 (4E-BP1). While Torin1 resulted in efficient dephosphorylation of S6K and 
bioRxiv preprint doi: https://doi.org/10.1101/2022.01.17.476702; this version posted January 18, 2022. The copyright holder for this preprint (which was not certified by peer review) is the author/funder, who has granted bioRxiv a license to display the preprint in perpetuity. It is made available under aCC-BY-NC-ND 4.0 International license.

4E-BP1, these drugs had no effect on phosphorylation of S6K and 4E-BP1 (Fig 3G-H), suggesting that their mode of action is mTOR independent. 


\section{Discussion}

Autophagy research has rapidly evolved and interest has heightened on examining the potential of novel/existing drugs to modulate autophagy [15]. Autophagy modulation is likely to have therapeutic benefits in several disease conditions [16]. Conversely, several FDA-approved drugs could be autophagy modulators but have not been studied from this perspective.

In our current study, we developed a reporter cell line for high throughput measurements of autophagy flux, and identified several known and novel autophagy modulators in the Spectrum collection library of 2560 compounds. Our primary screening and validation showed that three FDA-approved drugs that are widely used in skin-care preparations: avobenzone, guaiazulene, and tioxolone can efficiently induce autophagy flux in an mTOR independent manner.

Avobenzone (butyl methoxydibenzoylmethane; parsol 1789) is an ultraviolet A absorber widely used in sunscreens [17-23]. Studies have shown that avobenzone can efficiently penetrate the stratum corneum and viable epidermis [24]. It has been shown to protect against UVA-induced melanogenesis through indirect regulatory effect on the Nrf2-ARE pathway [25]. It has also been shown to inhibit the proliferation of human trophoblasts, enhance Akt and ERK1/2 activity and induce mitochondrial membrane depolarization [26]. In normal human epidermal keratinocytes, it can downmodulate lipid metabolism, and Peroxisome proliferator-activated receptor-gamma signaling [27], and can induce keratinocyte derived Vascular endothelial growth factor production [28]. Its autophagy inducing properties have not been described till date, however based on the abovementioned studies, it is likely that autophagy is likely to play an important role in the mode of action of avobenzone. 
Guaiazulene (1,4-dimethyl-7-isopropylazulene), is a natural azulenic compound widely used in cosmetic and health-care products and in pharmaceutical preparations such as creams and toothpastes $[29,30]$. Its early use was as an ophtlamic drug, and later it became a popular skin conditioning agent. The drug was also shown to be promising for the treatment of gastritis and peptic ulcers [31]. Studies have shown that it can act in a synergic manner with diclofenac and can have therapeutic advantages for the clinical treatment of inflammatory pain [32]. It has also been shown to have anti-cancer properties [33]. A recent study showed that guaiazulene inhibited Akt/mTOR signalling and induced autophagosome formation [34]

Tioxolone (6-Hydroxy-2H-1,3-benzoxathiol-2-one) is used for cosmetics products such as hair shampoos, skin cleansers and acne treatment products [35]. It has anti-fungal, antibacterial, anti-inflammatory and anti-tumorigenic effects, and has been shown to be an inhibitor of human carbonic anhydrase II [36, 37]. Tincture of thioxolone plus benzoxonium chloride was shown to be useful for the treatment of cutaneous leishmaniasis [38].

In summary, our high throughput screening approach has identified several novel autophagy inducers and inhibitors. We also validate that three widely used drugs in skincare products are potent autophagy inducers. Further studies will highlight if the autophagy inducing properties of these drugs govern their mode of action. 


\section{Conflict of interest}

The authors have no conflict of interest to declare

\section{Acknowledgements}

High content screening was performed at the Advanced Technology Platform Centre (ATPC)

at RCB. We are thankful to Dr. Nirpendra Singh and Ashish Pandey for technical support. All

Virology lab members are acknowledged for their support.

\section{Funding}

This work was supported by a grant from DBT BT/PR27875/Med/29/1302/2018, and from DBT intra-mural funds to RCB. SKP is supported by a DBT-SRF fellowship. 


\section{References:}

1. Mizushima, N., B. Levine, A.M. Cuervo, et al., Autophagy fights disease through cellular self-digestion. Nature, 2008. 451(7182): p. 1069-75.

2. Vakifahmetoglu-Norberg, H., H.G. Xia and J. Yuan, Pharmacologic agents targeting autophagy. J Clin Invest, 2015. 125(1): p. 5-13.

3. Choi, A.M., S.W. Ryter and B. Levine, Autophagy in human health and disease. N Engl J Med, 2013. 368(7): p. 651-62.

4. Klionsky, D.J., A.K. Abdel-Aziz, S. Abdelfatah, et al., Guidelines for the use and interpretation of assays for monitoring autophagy (4th edition)(1). Autophagy, 2021. 17(1): p. 1-382.

5. Mizushima, N., T. Yoshimori and B. Levine, Methods in mammalian autophagy research. Cell, 2010. 140(3): p. 313-26.

6. Kaizuka, T., H. Morishita, Y. Hama, et al., An Autophagic Flux Probe that Releases an Internal Control. Mol Cell, 2016. 64(4): p. 835-849.

7. Chauhan, S., Z. Ahmed, S.B. Bradfute, et al., Pharmaceutical screen identifies novel target processes for activation of autophagy with a broad translational potential. Nat Commun, 2015. 6: p. 8620.

8. Li, Y., S. McGreal, J. Zhao, et al., A cell-based quantitative high-throughput image screening identified novel autophagy modulators. Pharmacol Res, 2016. 110: p. 3549.

9. Zhang, L., J. Yu, H. Pan, et al., Small molecule regulators of autophagy identified by an image-based high-throughput screen. Proc Natl Acad Sci U S A, 2007. 104(48): p. 19023-8. 
10. Kimura, S., T. Noda and T. Yoshimori, Dissection of the autophagosome maturation process by a novel reporter protein, tandem fluorescent-tagged LC3. Autophagy, 2007. 3(5): p. 452-60.

11. Reya, T., A.W. Duncan, L. Ailles, et al., A role for Wnt signalling in self-renewal of haematopoietic stem cells. Nature, 2003. 423(6938): p. 409-14.

12. Zhang, P., C. Rausch, F.D. Hastert, et al., Methyl-CpG binding domain protein 1 regulates localization and activity of Tet1 in a CXXC3 domain-dependent manner. Nucleic Acids Res, 2017. 45(12): p. 7118-7136.

13. Geng, J. and D.J. Klionsky, Direct quantification of autophagic flux by a single molecule-based probe. Autophagy, 2017. 13(4): p. 639-641.

14. Huang, L., Q. Fu, J.-M. Dai, et al., High-content screening of diterpenoids from Isodon species as autophagy modulators and the functional study of their antiviral activities. Cell Biology and Toxicology, 2021.

15. Kataura, T., E. Tashiro, S. Nishikawa, et al., A chemical genomics-aggrephagy integrated method studying functional analysis of autophagy inducers. Autophagy, 2021. 17(8): p. 1856-1872.

16. Yao, R.Q., C. Ren, Z.F. Xia, et al., Organelle-specific autophagy in inflammatory diseases: a potential therapeutic target underlying the quality control of multiple organelles. Autophagy, 2021. 17(2): p. 385-401.

17. Callen, J.P., D.E. Roth, C. McGrath, et al., Safety and efficacy of a broad-spectrum sunscreen in patients with discoid or subacute cutaneous lupus erythematosus. Cutis, 1991. 47(2): p. 130-2, 135-6. 
18. Hanson, K.M. and R.M. Clegg, Bioconvertible vitamin antioxidants improve sunscreen photoprotection against UV-induced reactive oxygen species. J Cosmet Sci, 2003. 54(6): p. 589-98.

19. Chatelain, E. and B. Gabard, Photostabilization of butyl methoxydibenzoylmethane (Avobenzone) and ethylhexyl methoxycinnamate by bis-ethylhexyloxyphenol methoxyphenyl triazine (Tinosorb S), a new UV broadband filter. Photochem Photobiol, 2001. 74(3): p. 401-6.

20. Gonzaga, E.R., Role of UV light in photodamage, skin aging, and skin cancer: importance of photoprotection. Am J Clin Dermatol, 2009. 10 Suppl 1: p. 19-24.

21. Wang, S.Q., J.W. Stanfield and U. Osterwalder, In vitro assessments of UVA protection by popular sunscreens available in the United States. J Am Acad Dermatol, 2008. 59(6): p. 934-42.

22. Beasley, D.G. and T.A. Meyer, Characterization of the UVA protection provided by avobenzone, zinc oxide, and titanium dioxide in broad-spectrum sunscreen products. Am J Clin Dermatol, 2010. 11(6): p. 413-21.

23. Kockler, J., S. Robertson, M. Oelgemöller, et al., Butyl methoxy dibenzoylmethane. Profiles Drug Subst Excip Relat Methodol, 2013. 38: p. 87-111.

24. Hayden, C.G., S.E. Cross, C. Anderson, et al., Sunscreen penetration of human skin and related keratinocyte toxicity after topical application. Skin Pharmacol Physiol, 2005. 18(4): p. 170-4.

25. Chaiprasongsuk, A., T. Onkoksoong, T. Pluemsamran, et al., Photoprotection by dietary phenolics against melanogenesis induced by UVA through Nrf2-dependent antioxidant responses. Redox Biol, 2016. 8: p. 79-90. 
26. Yang, C., W. Lim, F.W. Bazer, et al., Avobenzone suppresses proliferative activity of human trophoblast cells and induces apoptosis mediated by mitochondrial disruption. Reprod Toxicol, 2018. 81: p. 50-57.

27. Ahn, S., S. An, M. Lee, et al., A long-wave UVA filter avobenzone induces obesogenic phenotypes in normal human epidermal keratinocytes and mesenchymal stem cells. Arch Toxicol, 2019. 93(7): p. 1903-1915.

28. Bae, O.N., S. Ahn, S.H. Jin, et al., Chemical allergens stimulate human epidermal keratinocytes to produce lymphangiogenic vascular endothelial growth factor. Toxicol Appl Pharmacol, 2015. 283(2): p. 147-55.

29. Fiori, J., R. Gotti, A. Albini, et al., Study on the photostability of guaiazulene by highperformance liquid chromatography/mass spectrometry and gas chromatography/mass spectrometry. Rapid Commun Mass Spectrom, 2008. 22(17): p. $2698-706$.

30. Fiori, J., R. Gotti, L. Valgimigli, et al., Guaiazulene in health care products: determination by GC-MS and HPLC-DAD and photostability test. J Pharm Biomed Anal, 2008. 47(4-5): p. 710-5.

31. Okabe, S., K. Takeuchi, Y. Mori, et al., [Effects of KT1-32 on acute gastric lesions and duodenal ulcers induced in rats]. Nihon Yakurigaku Zasshi, 1986. 88(6): p. 467-76.

32. Ortiz, M.I., E. Fernandez-Martinez, L.E. Soria-Jasso, et al., Isolation, identification and molecular docking as cyclooxygenase (COX) inhibitors of the main constituents of Matricaria chamomilla L. extract and its synergistic interaction with diclofenac on nociception and gastric damage in rats. Biomed Pharmacother, 2016. 78: p. 248-256. 
33. Teratani, M., S. Nakamura, H. Sakagami, et al., Antitumor Effects and Tumorspecificity of Guaiazulene-3-Carboxylate Derivatives Against Oral Squamous Cell Carcinoma In Vitro. Anticancer Res, 2020. 40(9): p. 4885-4894.

34. Ye, Q., L. Zhou, P. Jin, et al., Guaiazulene Triggers ROS-Induced Apoptosis and Protective Autophagy in Non-small Cell Lung Cancer. Front Pharmacol, 2021. 12: p. 621181.

35. Tao, Y., Q. Wang, K. Sun, et al., The molecular structure, spectroscopic features and electronic properties of tioxolone under the external electric field. Spectrochim Acta A Mol Biomol Spectrosc, 2020. 231: p. 118108.

36. Iyer, R., A.A. Barrese, 3rd, S. Parakh, et al., Inhibition profiling of human carbonic anhydrase II by high-throughput screening of structurally diverse, biologically active compounds. J Biomol Screen, 2006. 11(7): p. 782-91.

37. Barrese, A.A., 3rd, C. Genis, S.Z. Fisher, et al., Inhibition of carbonic anhydrase II by thioxolone: a mechanistic and structural study. Biochemistry, 2008. 47(10): p. 317484.

38. Daie Parizi, M.H., M. Karvar, I. Sharifi, et al., The topical treatment of anthroponotic cutaneous leishmaniasis with the tincture of thioxolone plus benzoxonium chloride (Thio-Ben) along with cryotherapy: a single-blind randomized clinical trial. Dermatol Ther, 2015. 28(3): p. 140-6. 


\section{Figure Captions}

Figure 1. Development of GFP-LC3-RFP cell-line for autophagy flux assays. (A) Schematic representation of the autophagy flux measurement assay using the GFP-LC3-RFP fluorescent probe. (B) The stable A375 (GFP-LC3-RFP) clone was analysed by western blotting using GFP, LC3, RFP and GAPDH antibodies. Cleavage of the GFP-LC3 from the RFP fragment is observed. (C) A375 (GFP-LC3-RFP) cells were treated with DMSO or autophagy inducer Torin1 $(1 \mu \mathrm{M})$ or inhibitor Baf-A1 $(100 \mathrm{nM})$ for 6 h. Cells were imaged on a confocal microscope. Scale bar $20 \mu \mathrm{m}$. (D) GFP/RFP intensity ratios were measured from 20-30 cells from two independent coverslips and normalized to DMSO control.

Figure 2. High throughput imaging screen of Microsource spectrum library. (A) The Microsource spectrum library consisting of 2560 drugs were screened for the identification of autophagy flux modulators. A375 (GFP-LC3-RFP) cells were treated with Torin1 $(1 \mu \mathrm{M})$, Baf-A1 (100 nM) for $6 \mathrm{~h}$, or with the DMSO/drugs $(10 \mu \mathrm{M})$ for $12 \mathrm{~h}$ in biological duplicates or triplicates. Images were acquired using the Image Xpress high content imaging system, and representative images are shown. Scale bar $100 \mu \mathrm{m}$. (B) GFP/RFP ratios were measured and normalized to DMSO control. Values show relative GFP/RFP ratios from 10 independent experiments. (C-D) Venn diagram showing the categorical classification of different molecules identified as autophagy inducers (ratio <0.8) or inhibitors (ratio >1.2). Clustered heatmap showing GFP/RFP ratios of autophagy modulators identified in the screen.

Figure 3. Characterization of Avobenzone, Guaiazulene and Tioxolone as autophagy inducers. (A) Viability measurements of A375 cells treated with Avobenzone/ Guaiazulene/ Tioxolone $(10 \mu \mathrm{M})$ for $12 \mathrm{~h}$. (B-C) A375 (GFP-LC3-RFP) cells were treated with control drugs (Torin1/ Baf-A1) or Avobenzone/ Guaiazulene/ Tioxolone $(n=3)$, and images were acquired 
using the Image Xpress high content imaging system. The relative ratiometric quantitation of GFP/RFP is plotted in (C). (D-H) A375 cells were treated with the indicated drugs (10 $\mu \mathrm{M})$ for $12 \mathrm{~h}$, and protein extracts were analysed by western blotting with LC3 and GAPDH antibodies (D). Bar graph shows relative quantitation of LC3 expression levels normalized to GAPDH from three independent experiments (E). Cells were also treated with Baf-A1 (100 $\mathrm{nM}$ ) for $2 \mathrm{~h}$ before harvest and immunoblotted with LC3 and GAPDH antibodies. Increase in LC3-II levels on Baf-A1 treatment is indicative of functional autophagy flux. Numbers below the blot indicate ratio of LC3-II/GAPDH, calculated using Image J (F). (G-H) mTOR downstream signaling was checked by measuring the proteins level of $p-T 386 S 6 K 1$, S6K1, p4E-BP1(Thr37/46) and 4E-BP1 antibodies. Torin1 treatment was used as a positive control. Data is representative of three independent experiments. 


\section{Supplementary Data}

File S1: Autophagy flux primary screen data showing \% nuclei count and GFP/RFP ratios for the 2560 compounds of the Microsource spectrum library (Sheet: GFP,RFP ratios); list of novel and known autophagy inducers identified in the study. PMID's for the known autophagy inducers are indicated (Sheet: inducers); list of novel autophagy flux inhibitors identified (Sheet: inhibitors). 
A

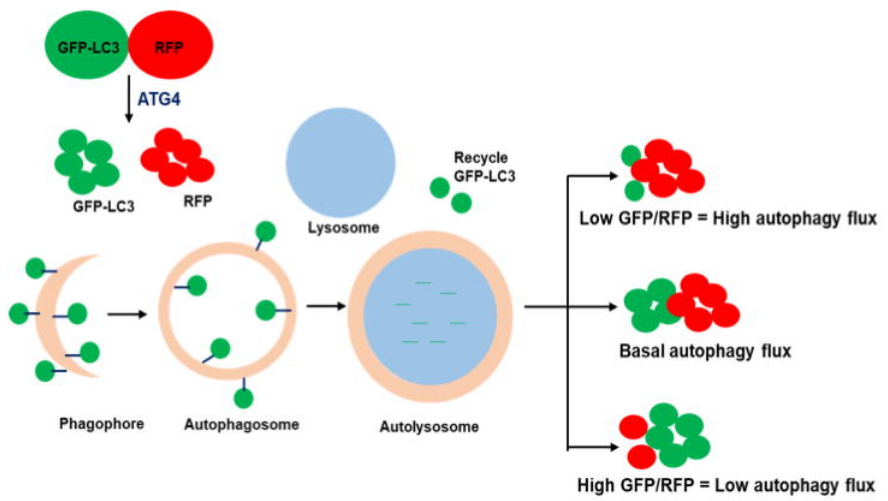

C

DAPI / GFP

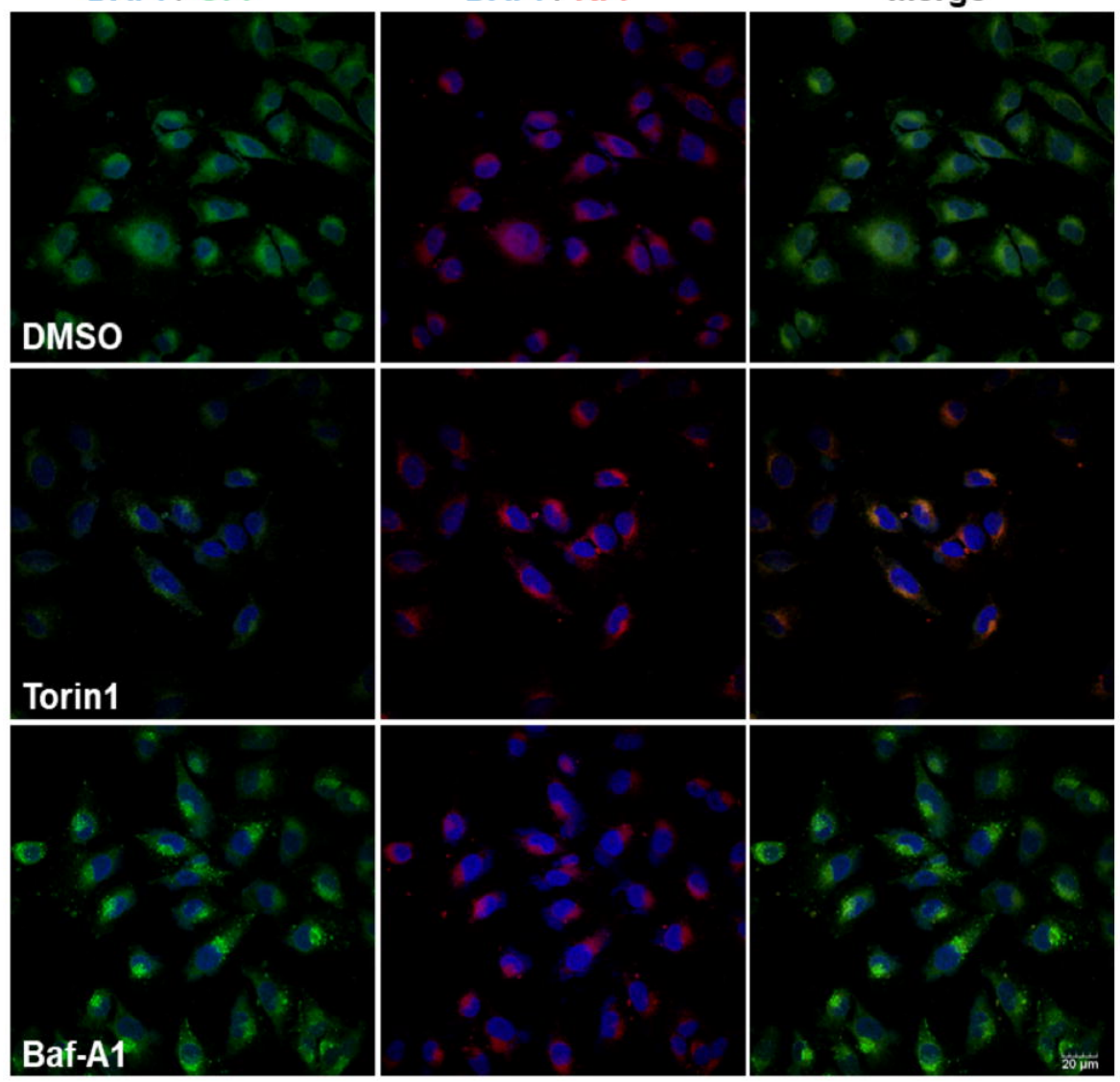

B

A375 (GFP-LC3-RFP)

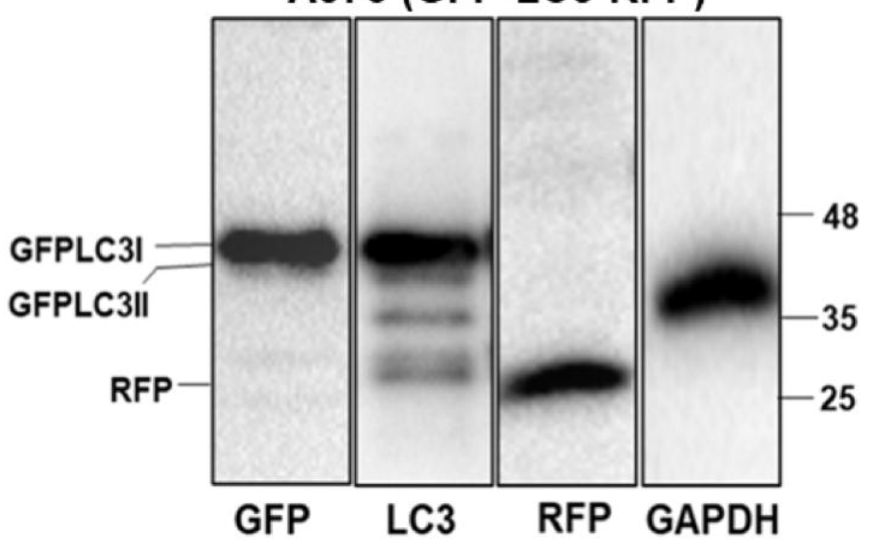

D

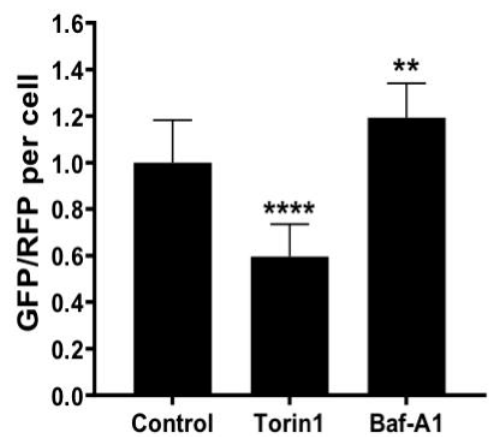


A

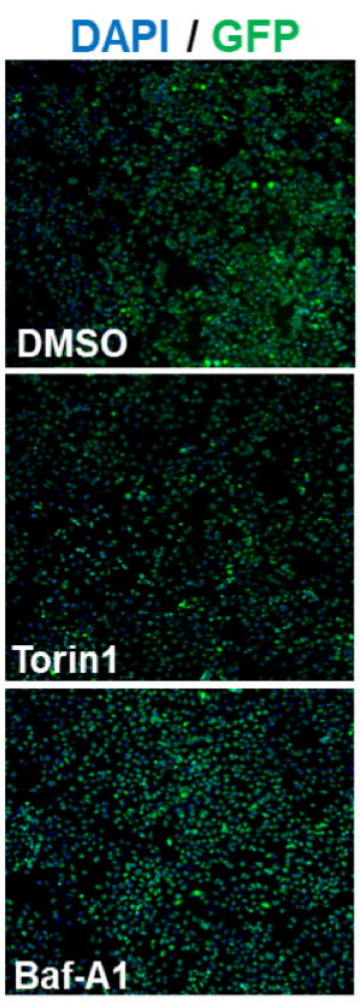

\section{DAPI / RFP}

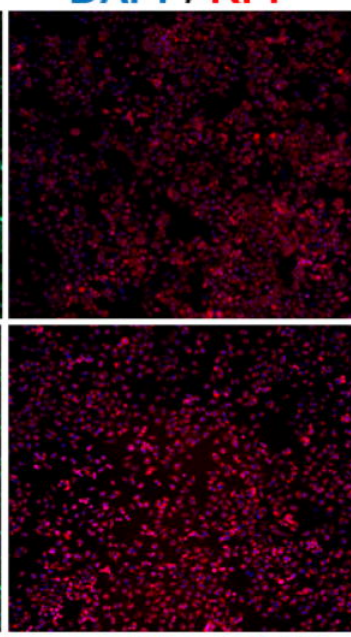

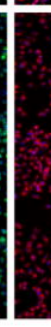

B

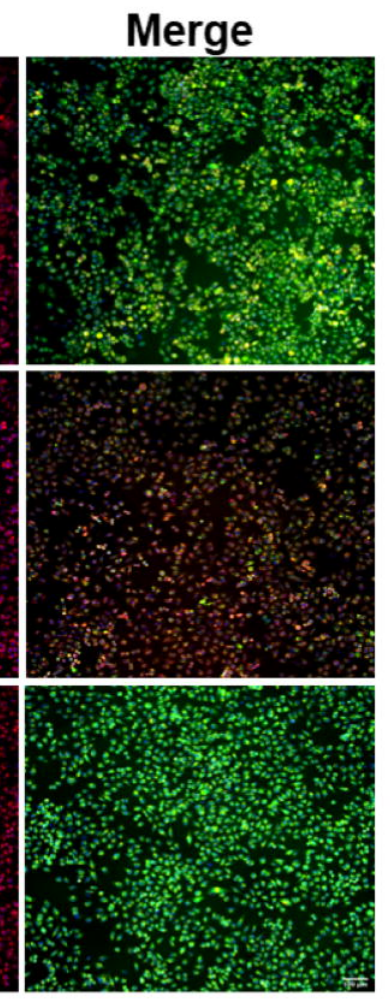

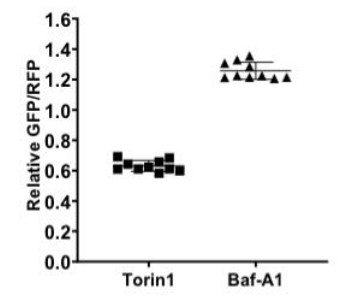

C

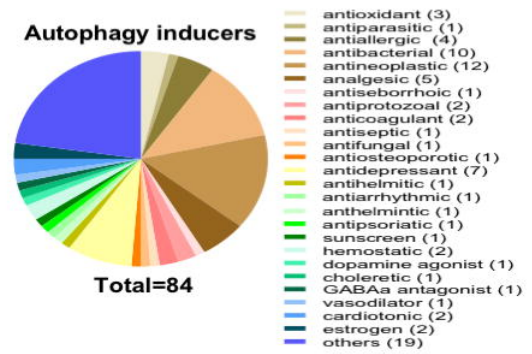

D

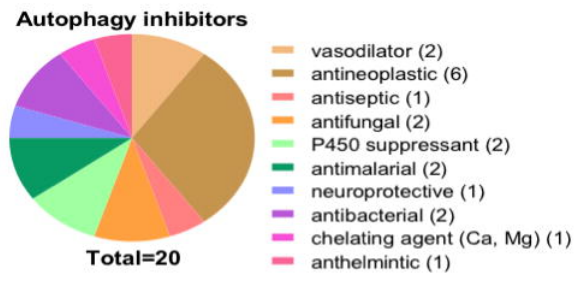

E

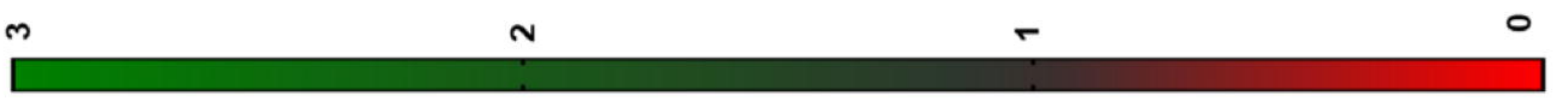

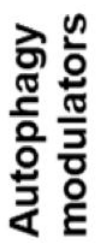

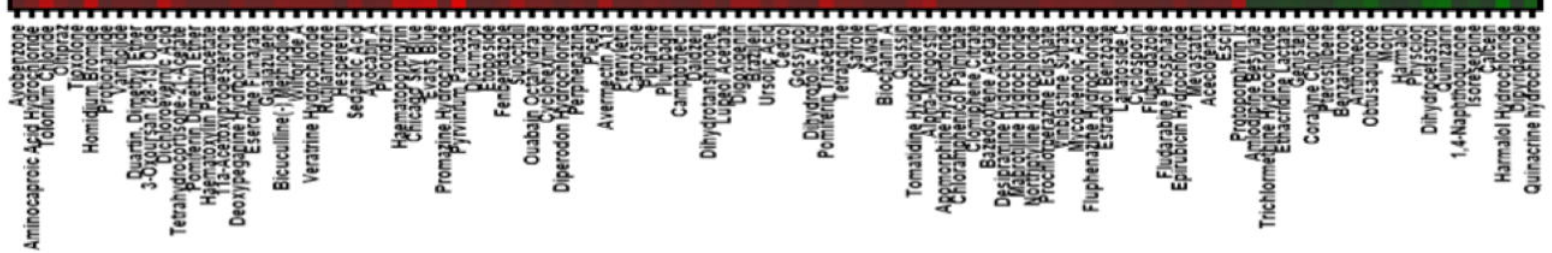




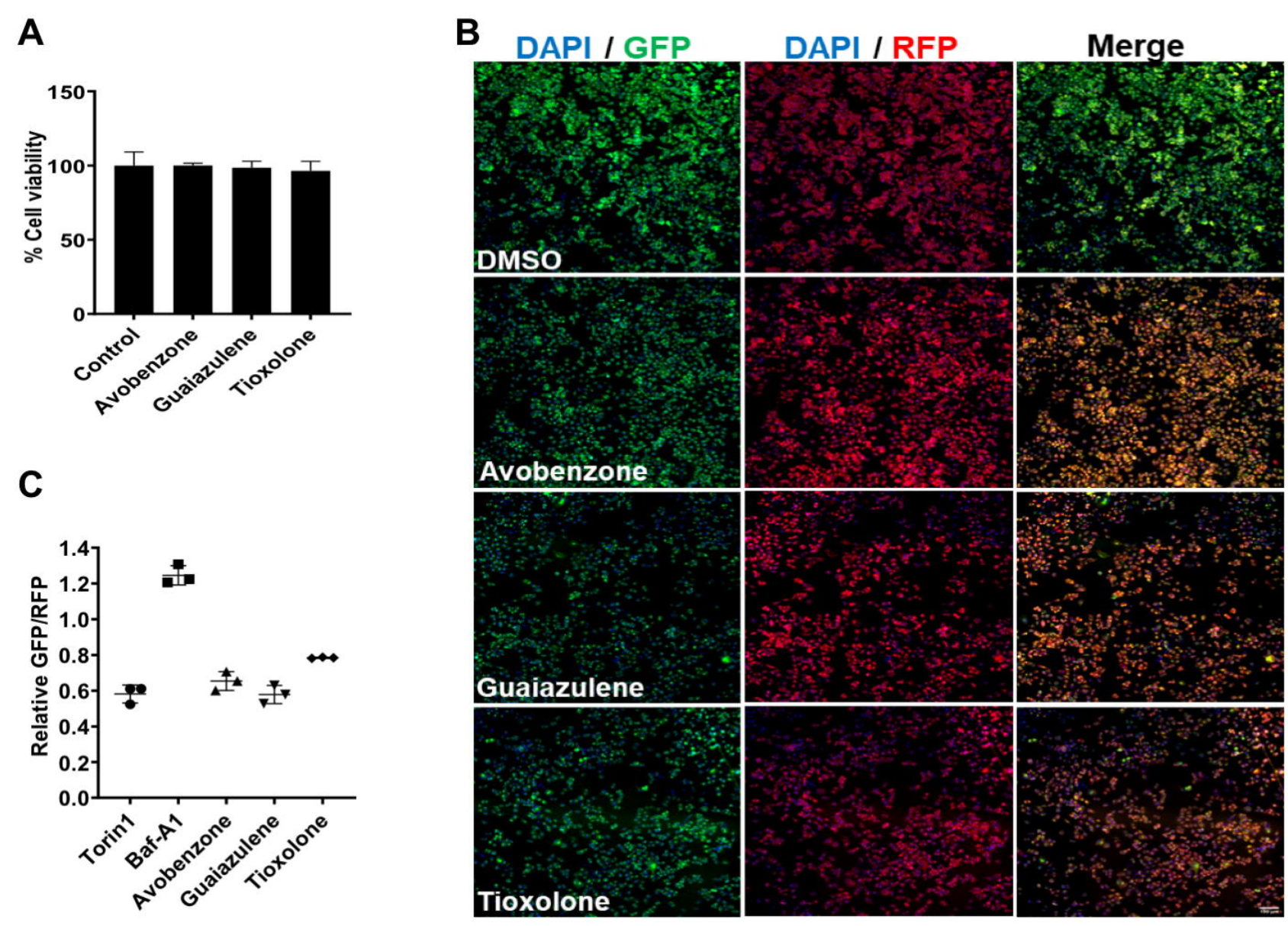

D

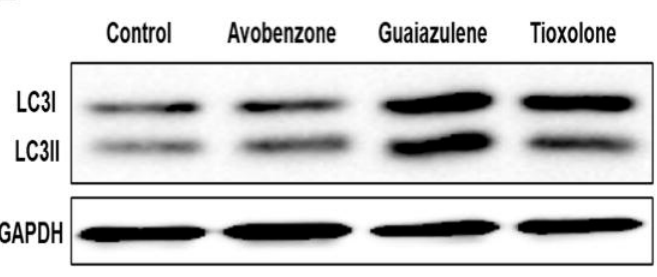

F

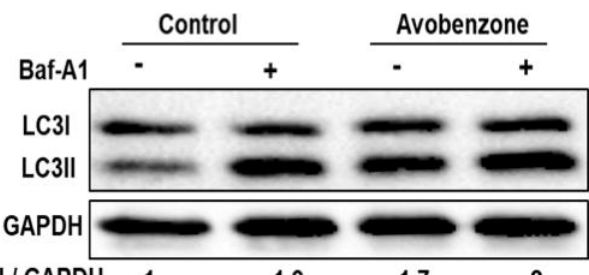

G

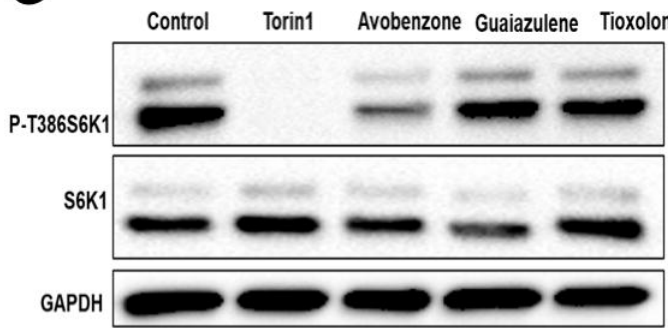$$
\text { C3II }
$$$$
1.9
$$$$
1.7
$$$$
2
$$

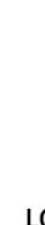

E

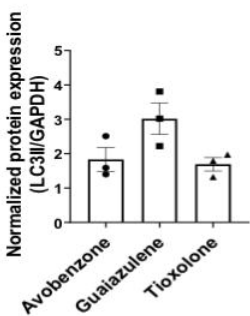

